\title{
Caracterização de compósitos particulados de polietileno de alta densidade/pó de concha de molusco
}

\section{Characterization of the particulate composites of high density polyethylene/mollusk shell powder}

\author{
Helen Cristina Torrano Firmino ${ }^{1}$, Thais Fernandes das Chagas ${ }^{1}$, \\ Patrícia Maria Alves de Melo $^{2}$, Lucineide Balbino da Silva ${ }^{2}$
}

\author{
${ }^{1}$ Universidade Federal da Paraíba-Departamento de Engenharia de Materiais CP: 58051 900, João Pessoa, PB \\ e-mail: hellentorrano@hotmail.com; thais_chagaspb@yahoo.com.br \\ ${ }^{2}$ Universidade Federal da Paraíba-Programa de Pós Graduação em Ciência e Engenharia de Materiais-PPCEM. CP: \\ 58051 900, João Pessoa-PB \\ e-mail: paty.melo@hotmail.com; Lucineide@ct.ufpb.br
}

\section{RESUMO}

O objetivo deste trabalho foi investigar as propriedades térmicas, mecânicas e termo dinâmico mecânicas de compósitos de polietileno de alta densidade (PEAD) com a incorporação do pó de conchas de moluscos (5, 10 e $20 \%$ em peso). A matriz pura e seus compósitos foram misturados em um misturador interno e em seguida moldados por compressão. A incorporação do pó de concha de molusco diminuiu o índice de fluidez do PEAD em uma faixa de 23 a 53\%, já a cristalinidade da matriz praticamente não foi alterada. O pó da concha possui $97,53 \%$ de $\mathrm{CaO}$, tendo estrutura cristalina constituída por aragonita e calcita, ambos polimorfos do carbonato de cálcio. $\mathrm{O}$ estudo granulométrico mostrou que o pó da concha apresentou uma distribuição larga do tamanho das partículas, sendo também confirmada na análise da superfície de fratura dos compósitos por microscopia eletrônica de varredura. Os aspectos morfológicos mostram que nenhuma adesão foi observada entre as partículas e o PEAD. A adição da concha causou um aumento na rigidez da matriz e como também diminui a capacidade de amortecimento da matriz. A adição de $10 \%$ deslocou a temperatura de transição vítrea $(\mathrm{Tg})$ do PEAD em $6{ }^{\circ} \mathrm{C}$, enquanto o compósito com $20 \%$ do pó de concha não apresentou essa transição.

Palavras chaves: PEAD, concha de molusco, compósito, moldagem por compressão, análise térmica.

\section{ABSTRACT}

This work deals with an investigation of thermal, mechanical and dynamic mechanical properties of composites of high density polyethylene(HDPE) with addition of mollusk shell powder (5, 10 and $20 \%$ wt). The pure matrix and its composites were first mixed in an internal mixer and then molded by compression. The mollusk shell powder incorporation decreased the melt index within a range of $23 \%$ to $53 \%$ whereas no relevant changes in crystallinity of matrix were observed. The shell powder was composed by $97.53 \%$ of $\mathrm{CaO}$ and its crystalline structure was constituted of calcite and aragonite, both polymorphs of calcium carbonate. The granulometric study showed a large distribution of particle size to the mollusk shell powder, which was also confirmed by the scanning electron microscopy (SEM) analysis of the fracture surface of the composites. The morphological aspects of the composites showed a poor adhesion between HDPE matrix and shell powder. The adding of filler increased the matrix rigidity and decreased its damping capacity. The incorporation of $10 \% \mathrm{wt}$ mollusk shell powder shifted the glassy transition temperature (Tg) of the PEAD in 6 ${ }^{\circ} \mathrm{C}$, while the composite with $20 \%$ wt did not show this transition temperature.

Keywords: HDPE, mollusk shell powder, composite, molding compression, thermal analysis.

\section{INTRODUÇÃO}

A preocupação com o meio ambiente tem sido constante nos dias atuais e pesquisas, que venham a contribuir com a diminuição dos danos causados ao ambiente, têm sido bastante motivadas. Na costa Brasileira, em especial na região litorânea de João Pessoa, a atividade de comunidades marisqueiras produz cascas de moluscos, especificamente das espécies Anomalocardia brasiliana e Tivela mactroides. A exploração destes 
moluscos bivalves representa o sustento destas comunidades que sobrevivem dos recursos oferecidos pelo estuário de alguns rios, que desembocam no litoral paraibano. As conchas não têm valor comercial, sendo difícil o descarte.

As cargas minerais são bastante utilizadas como material de reforço em matrizes poliméricas termoplásticas por promover muitos benefícios econômicos e mecânicos as mesmas, entre elas, o carbonato de cálcio $\left(\mathrm{CaCO}_{3}\right)$ é um mineral muito utilizado. A utilização do pó da concha de molusco em compósitos poliméricos vislumbra uma possibilidade de reutilização da mesma com potencial para modificar o polietileno de alta densidade (PEAD) positivamente. Isto porque sua estrutura cristalina e inclusive as de diversos outros tipos de moluscos são constituídas por calcita ou aragonita, polimorfos do $\mathrm{CaCO}_{3}$. Além da estrutura cristalina, a concha também possui uma matriz de macromoléculas orgânicas nanométricas dispostas entre as camadas inorgânicas, se arranjando em uma arquitetura estrutural complexa, resultando em materiais leves, com alta performance mecânica; por exemplo, a tenacidade a fratura da concha excede de duas a três ordens de magnitude daquela do cristal único do mineral de carbonato de cálcio puro [1-8].

Sabe-se que ao adicionar cargas a polímeros semicristalinos, todas as suas propriedades estão sujeitas às mudanças e dependem do tipo que é adicionada ao polímero, por exemplo, dependem do tamanho de suas partículas, da forma e da rigidez e também da sua concentração na matriz polimérica e do tipo de interação entre elas próprias e entre elas e o polímero. Por exemplo, as partículas de carbonato de cálcio com razão de aspecto próxima a unidade são esperadas modificar a viscosidade do polímero fundido. Além disso, a sua boa condutividade térmica contribui com a homogeneidade do fundido e com a boa dispersão dela na matriz polimérica. As cargas minerais são adicionadas aos polímeros semicristalinos em uma faixa de composição de 10-40\% em peso, causando diversos melhoramentos nas propriedades mecânicas das matrizes poliméricas: no módulo sob tração e sob flexão, na resistência a tração e ao impacto, na capacidade do polímero em retardar a propagação de chama e na sua resistência a deformação térmica ou distorção. Além disso, também diminui a expansão térmica do polímero e o seu encolhimento dentro da cavidade do molde, durante a etapa de processamento. O módulo da matriz aumenta com o aumento na fração de volume da carga, resultando em uma restrição na mobilidade das cadeias poliméricas e consequentemente na flexibilidade da matriz. A tensão de escoamento é fortemente influenciada pelo tamanho de partícula da carga, pela sua interação interfacial com o polímero e sua dispersão na matriz polimérica [1, 9-10].

O objetivo deste trabalho foi obter compósitos poliméricos com a incorporação de pó de concha de molusco após a mistura em um misturador interno, seguido por moldagem por compressão e realizar análises térmicas, , morfológicas e termo-dinâmico-mecânicas dos compósitos com concentrações do pó de concha de 5,10 e $20 \%$ em peso.

\section{MATERIAIS E MÉTODOS}

Foi utilizado o Polietileno de Alta Densidade (PEAD) fornecido pela empresa Eteno-Empresa de Termoplásticos Ltda., localizada em Recife-PE. A concha do molusco foi fornecida pela comunidade do litoral paraibano, sendo utilizada como carga particulada após a sua moagem e classificação granulométrica.

\subsection{Moagem e Classificação Granulométrica das Conchas}

As conchas foram lavadas e secas ao sol e em seguida moídas em um moinho de bolas e a granulometria foi classificada em peneira ABNT de malha 200 mesh $(0,074 \mathrm{~mm})$. O tamanho das partículas da concha foi determinado usando um equipamento CILAS em uma faixa de tamanho de 0,04-500 microns/100 classes, no modo úmido usando água destilada.

\subsection{Caracterização Microestrutural da Concha Moída}

A difração de raios $X$ foi realizada utilizando um difratômetro D5000 fabricado pela SIEMENS. Nesse ensaio se utilizou um tubo de $\mathrm{Cu}$ com comprimento de onda de $1,5406 \AA$, com tensão de $40 \mathrm{kV}$, corrente de $30 \mathrm{~mA}$. A análise química da composição da concha foi obtida em um espectrômetro de fluorescência por difração de raiosX fabricado pela Lab Center, modelo Sequential X Ray Fluorescence Spectrometer.

\subsection{Análise do Índice de Fluidez (MFI)}

O ensaio de Índice de Fluidez (MFI) do PEAD puro e dos seus compósitos foi realizado em um plastômetro Melt Flow Modular Line da Ceast, segundo a Norma ASTM D1238, a uma temperatura de $190{ }^{\circ} \mathrm{C}$ e peso de 2,16 Kg. O índice de fluidez foi determinado após o PEAD e os compósitos serem misturados no misturador interno e moídos em um moinho de facas. 


\subsection{Moldagem por Compressão dos Compósitos}

Inicialmente, o PEAD e seus compósitos, nas concentrações de 5, 10 e 20\% em peso do pó de concha de molusco, foram processados em um misturador interno Polylab OS da HAAKE, a uma temperatura de 180 ${ }^{\circ} \mathrm{C}$, rotação de $100 \mathrm{rpm}$ e tempo de mistura de $10 \mathrm{~min}$. Após a etapa de processamento, as amostras foram moídas em um moinho de facas, adquirindo o formato de pó e antes da moldagem foram secos durante 2 horas em uma estufa para a retirada de umidade, a $70{ }^{\circ} \mathrm{C}$. Em seguida os corpos de prova foram moldados por compressão em uma prensa hidráulica, a uma temperatura de $170{ }^{\circ} \mathrm{C}$. Inicialmente a amostra ficou durante 3 min sem aplicação de pressão e em seguida uma pressão de 3 toneladas foi aplicada durante 5 min.

O objetivo da moldagem por compressão foi preparar corpos de prova no formato de placas, para a caracterização dinâmico termo mecânica e análise da superfície de fratura.

\subsection{Análise Térmica dos Compósitos}

Por meio da análise de calorimetria exploratória diferencial (DSC) foi possível verificar se ocorreram mudanças nas transições ocorridas no PEAD com a adição do pó de concha do molusco. As análises foram realizadas em um DSC-60, Marca Shimadzu, a uma taxa de $10{ }^{\circ} \mathrm{C} / \mathrm{min}$. Primeiro as amostras foram submetidas ao aquecimento a uma taxa de $10{ }^{\circ} \mathrm{C} / \mathrm{min}$ até $200{ }^{\circ} \mathrm{C}$, mantidas por 3 min nessa mesma temperatura para eliminar a história térmica das amostras, em seguida foi realizada uma varredura de resfriamento e em seguida aquecida novamente a $10{ }^{\circ} \mathrm{C} / \mathrm{min}$ até $200{ }^{\circ} \mathrm{C}$. Os valores de temperatura de fusão (Tf) e entalpia de fusão $\left(\Delta \mathrm{H}_{\mathrm{f}}\right)$ das amostras foram determinadas no segundo aquecimento.

O grau de cristalinidade foi calculado através da seguinte fórmula:

$$
X_{c}(\%)=\frac{\Delta H_{f}}{\left(1-W_{\mathrm{f}}\right) \cdot \Delta H_{100 \%}}
$$

Onde $\mathrm{X}_{\mathrm{c}}$ é o grau de cristalinidade, $\Delta \mathrm{H}_{\mathrm{f}}$ é a entalpia de fusão do PEAD e dos compósitos obtidos através da análise do DSC, e $\Delta \mathrm{H}_{100 \%}$ é a entalpia de fusão para o PEAD $100 \%$ cristalino, cujo valor é igual a $291 \mathrm{~J} / \mathrm{g}[11]$.

A análise termogravimétrica forneceu informações se a adição da concha influenciou a temperatura de degradação térmica do PEAD. Para tanto foi utilizado um equipamento modelo DTG-60H, simultâneo DTATG. A análise foi realizada sob fluxo de argônio de $50 \mathrm{ml} / \mathrm{min}$, a fim de evitar a oxidação, a uma taxa de aquecimento de $10{ }^{\circ} \mathrm{C} / \mathrm{min}$. Em virtude da temperatura alta de degradação do pó da concha, a sua análise ocorreu em um intervalo de temperatura de $25{ }^{\circ} \mathrm{C}$ à $900{ }^{\circ} \mathrm{C}$. Para os compósitos se utilizou uma faixa de temperatura entre $50{ }^{\circ} \mathrm{C}$ e $400{ }^{\circ} \mathrm{C}$, devido ao interesse em se verificar apenas a degradação da matriz de PEAD.

\subsection{Análise Dinâmico Mecânica (DMA)}

O módulo dinâmico do PEAD puro e dos compósitos foi determinado em um Analisador Dinâmico Mecânico DMA Q800 da TA Instruments. Os ensaios foram conduzidos no modo de flexão em três pontos, a uma frequência de vibração constante de $1 \mathrm{~Hz}$, temperatura na faixa de $30-100{ }^{\circ} \mathrm{C}$ e taxa de aquecimento de 3 ${ }^{\circ} \mathrm{C} / \mathrm{min}$. As dimensões da amostra foram determinadas de acordo com a Norma ASTM D 5418-07.

\subsection{Análise da Morfologia por Microscopia Eletrônica de Varredura}

A morfologia das amostras foi avaliada após a fratura dúctil das mesmas. A análise foi realizada em um Microscópio Eletrônico de Varredura, marca LEO 1430. As superfícies de fratura das amostras foram metalizadas com ouro antes da investigação microscópica. A análise foi realizada por meio do sinal gerado pelos elétrons secundários.

\section{RESULTADOS E DISCUSSÕES}

\subsection{Análise microestrutural e granulométrica da concha moída}

A composição química da concha de molusco utilizada neste trabalho é apresentada na Tabela 1. Pode-se observar a maior predominância de óxido de cálcio nas conchas, no valor de 97,53\%, e também outros elementos (ferro, estrôncio e cromo), a níveis muito mais baixos. A concentração da sílica na concha é de 
$0,80 \%$, sua presença não é desejável, pois pode contribuir com a abrasividade da concha, causando danos nos equipamentos de processamento de polímeros, durante a fabricação do compósito polimérico.

Tabela 1: Resultados da Análise Química das conchas por Fluorescência de Raios X.

\begin{tabular}{l|l}
\hline COMPOSTO & PORCENTAGEM (\%) \\
\hline $\mathrm{CaO}$ & 97,5372 \\
\hline $\mathrm{Fe}_{2} \mathrm{O}_{3}$ & 0,8697 \\
\hline $\mathrm{SiO}_{2}$ & 0,8029 \\
\hline $\mathrm{SrO}$ & 0,5300 \\
\hline $\mathrm{SO}_{3}$ & 0,1462 \\
\hline $\mathrm{Cr}_{2} \mathrm{O}_{3}$ & 0,1140 \\
\hline
\end{tabular}

A estrutura cristalina da concha foi avaliada por Difração de Raios X, conforme se observa na Figura 1. As posições dos picos, em $2 \theta$ igual a $25^{\circ}$ e próximo a $30^{\circ}$ correspondem à fase cristalina de aragonita $\mathrm{e}$ calcita, respectivamente. $\mathrm{O} \mathrm{CaCO}_{3}$ é o segundo mineral mais abundante na crosta terrestre, excedido apenas pelo quartzo. Duas formas cristalinas mais conhecidas para o carbonato de cálcio são a calcita e a aragonita, uma é mais abundante, cristalizando no sistema triclínico e a outra é menos abundante, cristalizando no sistema ortogonal, respectivamente. Estes picos para ambas as formas cristalinas também foram observados por outros autores 0 . Portanto, pode-se concluir que a concha seja composta essencialmente por óxido de cálcio, cuja formação ocorreu ao longo do tempo. Portanto, a utilização do pó de concha de molusco como carga particulada mineral em compósito polimérico pode ser bastante promissora.11

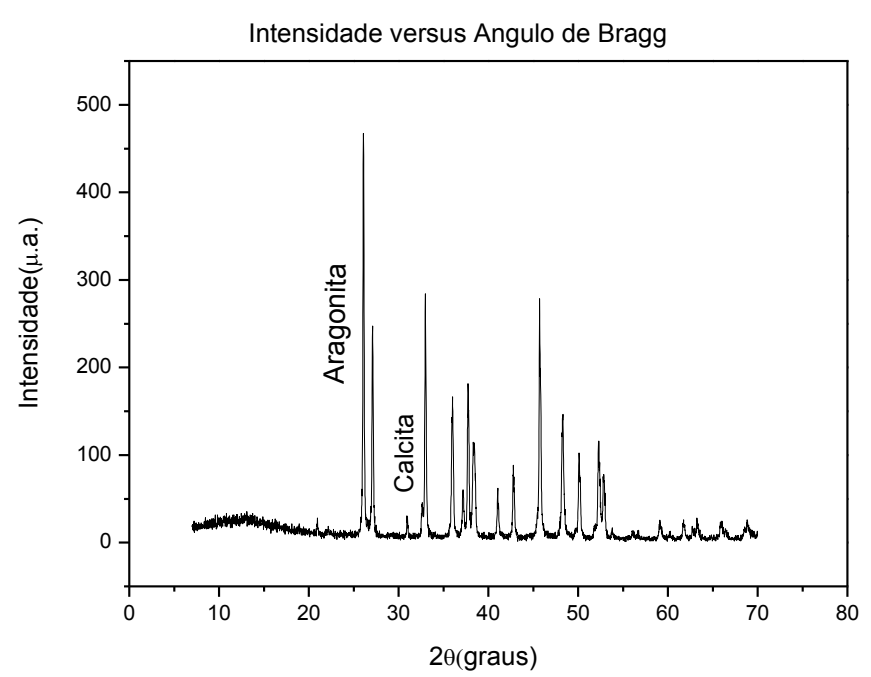

Figura 1: Resultados da Difração de Raios X da concha de molusco.

Em virtude da composição química da concha de molusco ser predominantemente composta por $\mathrm{CaO}$ e a sua estrutura cristalina ser composta por aragonita e calcita, ambas estruturas polimorfas do $\mathrm{CaCO}_{3}$, o pó da concha de molusco foi adicionada ao PEAD visando melhoria nas propriedades mecânicas da matriz.

Em termos da análise granulométrica conforme mostra a Figura 2, o pó da concha apresentou uma larga distribuição de tamanho de partículas com $10 \%$ dos diâmetros com tamanho de $0,80 \mu \mathrm{m}, 50 \%$ com 6,49 $\mu \mathrm{m}$ e $90 \%$ com $36,35 \mu \mathrm{m}$ que resultou em tamanho médio de tamanho de partículas igual a $12,51 \mu \mathrm{m}$.

\section{2 Índice de Fluidez (MFI)}

Os valores de MFI do PEAD puro e dos seus compósitos são mostrados na Tabela 2. Pode se observar que o MFI do PEAD puro foi maior do que os valores dos compósitos. A adição de $10 \%$ da concha diminuiu em $53 \%$ o índice de fluidez do PEAD, seguido pelo compósito com 5\% de concha. Já o MFI do compósito contendo $20 \%$ de concha, em relação aos demais compósitos, teve uma diminuição em torno de $23 \%$. A 
redução do MFI significa uma redução na fluidez e na processabilidade desses compósitos. As partículas da concha podem ter causado uma interrupção no polímero fundido ao ser extrudado no plastômetro, aumentando a viscosidade dos compósitos fundidos. Essa redução no fluxo do fundido pode ser atribuído ao fato das partículas da concha não sofrerem processo de fusão durante a extrusão, esse resultado concorda com Ali Syed e colaboradores [13] ao estudarem compósitos particulados de PEAD.

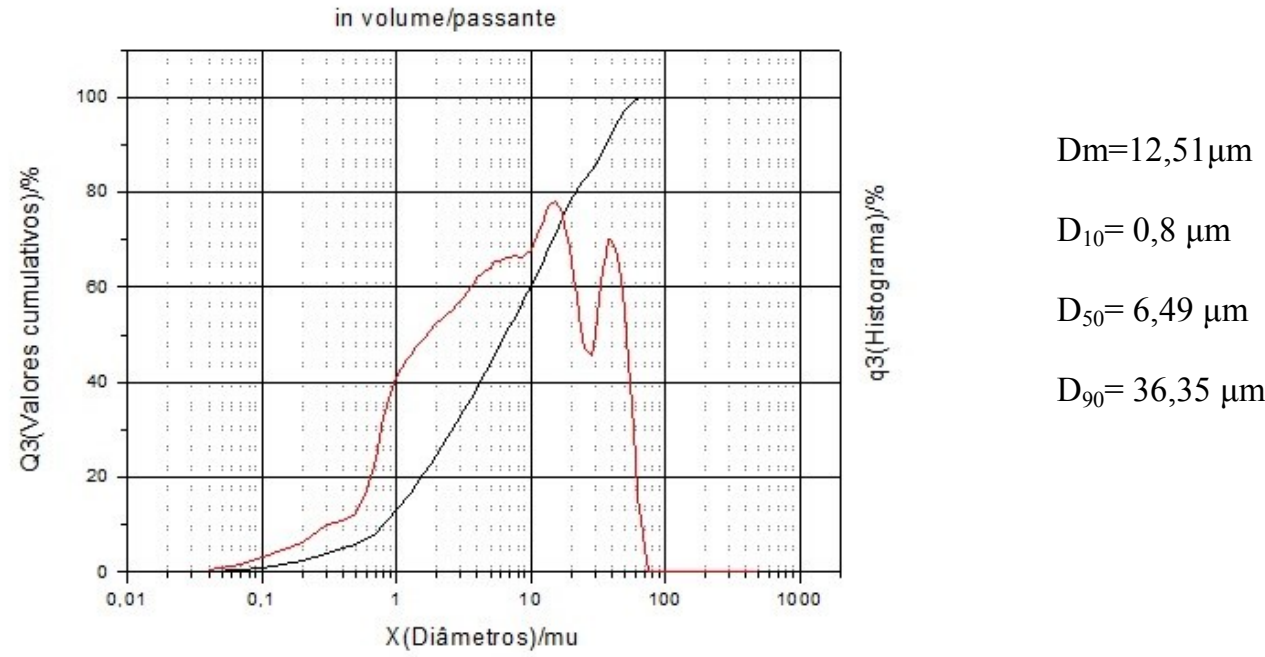

Figura 2: Resultados do tamanho de partículas do pó de concha de molusco.

A determinação do índice de fluidez foi importante para compreender a baixa processabilidade dos compósitos analisados nesse estudo. O fato dos compósitos terem mais baixa propriedade de fluxo do que o PEAD puro tornou a sua moldagem por compressão mais difícil, isto porque há a necessidade do preenchimento de regiões mais estreitas da cavidade do molde, utilizando as pressões baixas da moldagem por compressão. Portanto, a adição do pó da concha ao PEAD em concentrações superiores a $20 \%$ poderá comprometer o processamento desses compósitos, sendo necessário adicionar um aditivo de fluxo para melhorar a processabilidade desses compósitos.

Tabela 2: Índice de Fluidez (MFI) do PEAD puro e dos seus compósitos.

\begin{tabular}{l|l}
\hline AMOSTRA & ÍNDICE DE FLUIDEZ (G/10MIN) \\
\hline PEAD puro & $6,75(0,18)$ \\
\hline $95: 5$ & $4,06(0,19)$ \\
\hline $90: 10$ & $3,625(0,13)$ \\
\hline $8: 20$ & $5,17(0,14)$ \\
\hline
\end{tabular}

\subsection{Análise Térmica Calorimetria exploratória diferencial (DSC)}

A Figura 3 mostra o comportamento de fusão do PEAD puro e dos seus compósitos. A Figura 3a mostra as curvas do primeiro aquecimento até $200{ }^{\circ} \mathrm{C}$ e mantidas por 3 min para apagar a história térmica que as amostras foram submetidas previamente. Em seguida, as amostras foram resfriadas e novamente aquecidas até $200{ }^{\circ} \mathrm{C}$, conforme mostrado na Figura 3b. A temperatura de fusão e a entalpia de fusão das amostras foram obtidas a partir do segundo aquecimento. Pode se observar que a temperatura de fusão da matriz não foi alterada com a adição da concha, já o comportamento de fusão foi alterado, de modo que o pico de fusão do PEAD foi mais estrito do que os picos dos compósitos, podendo ser atribuído aos cristais mais perfeitos da matriz pura. O alargamento nos picos de fusão dos compósitos sugere que ocorreu alguma modificação na estrutura dos cristais do PEAD, o que pode estar relacionado aos cristais menos perfeitos ou ainda uma possível distribuição de tamanhos dos cristais do PEAD nos compósitos. 


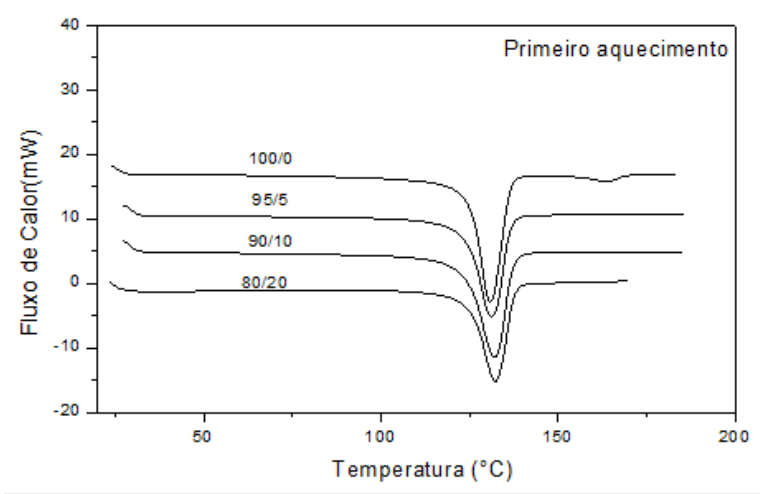

(a)

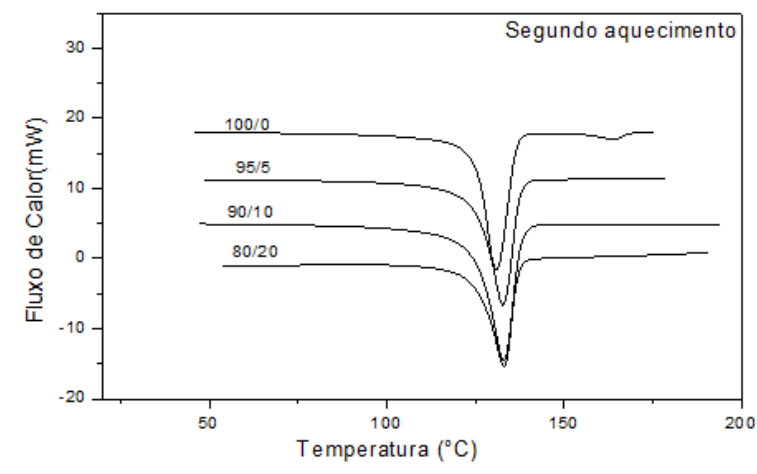

(b)

Figura 3: Curvas de DSC do PEAD puro e dos seus compósitos: a) Primeiro Aquecimento e b) Segundo Aquecimento.

Os valores da temperatura de fusão, a entalpia de fusão $\left(\Delta \mathrm{H}_{\mathrm{f}}\right)$ e o grau de cristalinidade $\left(\mathrm{X}_{\mathrm{c}}\right)$ do PEAD e dos seus compósitos são mostrados na Tabela 3. Pode se observar que a adição da concha não influenciou o grau de cristalinidade do PEAD, considerando as variações dos erros das medidas.

Tabela 3: Valores de entalpia de fusão, grau de cristalinidade e temperatura de fusão do PEAD puro e dos seus compósitos.

\begin{tabular}{l|l|l|l|l}
\hline & $\mathbf{1 0 0 / 0}$ & $\mathbf{9 5 / 5}$ & $\mathbf{9 0 / 1 0}$ & $\mathbf{8 0 / 2 0}$ \\
\hline$\Delta \mathbf{H}_{\mathbf{f}} \mathbf{( J / g )}$ & $140,16 \pm 3,55$ & $125,87 \pm 0,39$ & $109,72 \pm 9,80$ & $118,39 \pm 7,20$ \\
\hline $\mathbf{X}_{\mathbf{c}} \mathbf{( \% )}$ & $48,16 \pm 3,53$ & $45,86 \pm 0,38$ & $41,79 \pm 1,72$ & $50,85 \pm 2,71$ \\
\hline $\left.\mathbf{T}_{\mathbf{f}} \mathbf{(}^{\mathbf{0}} \mathbf{C}\right)$ & $136,18 \pm 3,34$ & $133,96 \pm 0,63$ & $133,24 \pm 0,33$ & $133,87 \pm 1,41$ \\
\hline
\end{tabular}

\subsection{Termogravimetria (TGA)}

Na Figura 4 são apresentadas as curvas de perda de massa em função da temperatura dos componentes puros e de seus compósitos. Na Tabela 4 são apresentados os dados obtidos a partir destas curvas e de suas derivadas (DTG). Pode se observar que ocorreu apenas um estágio de perda de massa para todas as amostras. O processo de degradação da concha é similar ao do carbonato de cálcio $\left(\mathrm{CaCO}_{3}\right)$, em que ocorre a formação de óxido de cálcio e dióxido de carbono, esse resultado está em concordância com [14]. Já os compósitos apresentaram dois picos de perda de massa, um relativo à decomposição do PEAD e o outro devido a decomposição da concha.

Pode se observar que as temperaturas de início da decomposição $\left(\mathrm{T}_{\text {Ińíio }}\right)$ dos compósitos foram maiores do que a do PEAD puro e o compósito contendo $20 \%$ do pó de concha apresentou maior valor, com uma diferença em torno de $37^{\circ} \mathrm{C}$ com relação a matriz pura. Este comportamento sugere que a adição das partículas da concha aumentou a temperatura de início da degradação do PEAD, ou seja, os compósitos foram mais estáveis. Já a temperatura da taxa máxima de perda de massa $\left(\mathrm{T}_{\max }\right)$ e a temperatura final de decomposição $\left(\mathrm{T}_{\text {Final }}\right)$ foram pouco alteradas com a adição da concha. Já a decomposição da concha ocorreu em torno de $743,64{ }^{\circ} \mathrm{C}$ e o teor de resíduo proveniente da sua degradação nos compósitos foi aumentada com a adição da mesma nos compósitos. 
Perda de M assa versus Temperatura

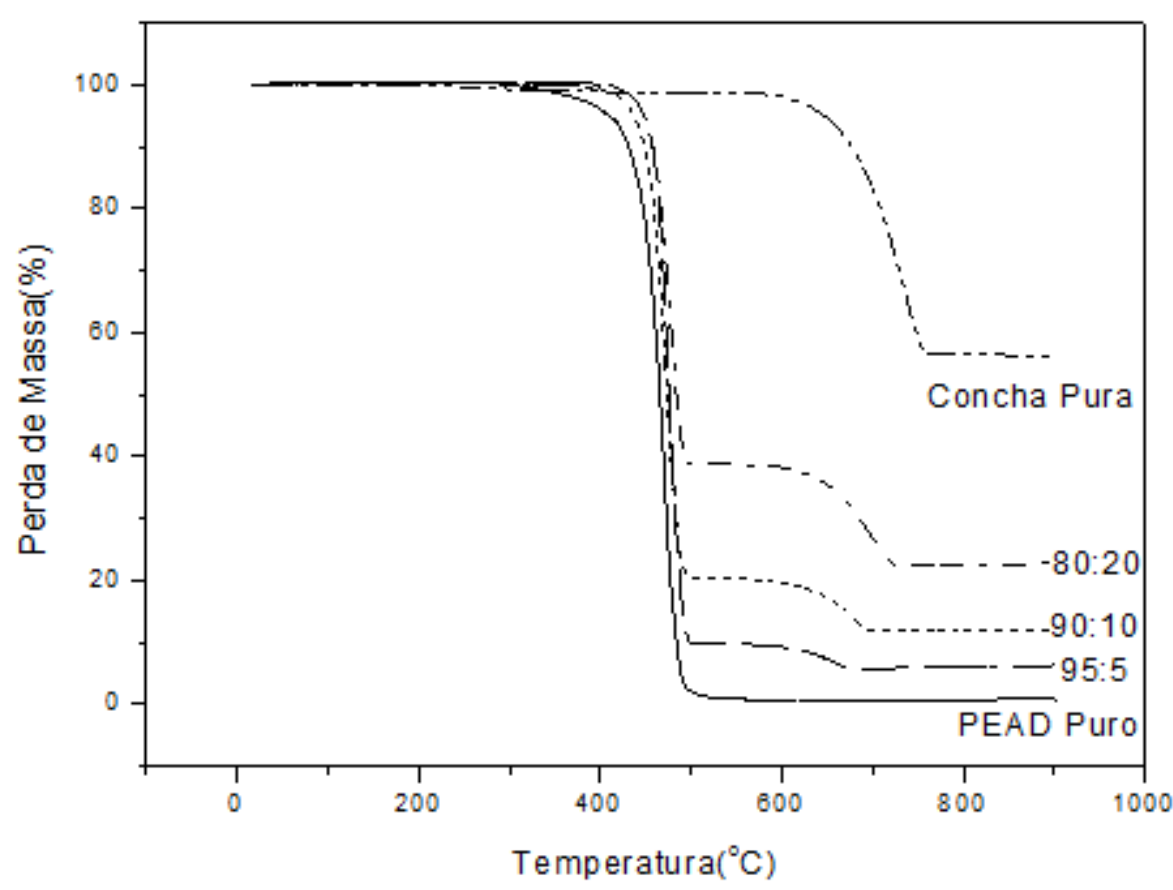

Figura 4: Curvas da Perda de massa em função da temperatura dos componentes puros e de seus compósitos.

Tabela 4: Dados de TGA e DTG dos componentes puros e seus compósitos.

\begin{tabular}{l|l|l|l|l}
\hline AMOSTRA & $\mathbf{T}_{\text {Início }}\left({ }^{\circ} \mathbf{C}\right)$ & $\mathbf{T}_{\max }\left({ }^{\circ} \mathbf{C}\right)$ & $\mathbf{T}_{\text {Final }}\left({ }^{\circ} \mathbf{C}\right)$ & RESíDUO (\%) \\
\hline $\mathbf{1 0 0} / \mathbf{0}$ & 423,73 & 477,67 & 494,23 & 0,45 \\
\hline $\mathbf{9 5} / \mathbf{5}$ & 444,30 & 469,00 & 494,23 & 6,52 \\
\hline $\mathbf{9 0} / \mathbf{1 0}$ & 456,85 & 477,67 & 481,68 & 11,61 \\
\hline $\mathbf{8 0} / \mathbf{2 0}$ & 461,12 & 485,95 & 490,22 & 21,76 \\
\hline Concha & 685,43 & 743,64 & 760,64 & 56,35 \\
\hline
\end{tabular}

\subsection{Análise morfológica (MEV)}

Os compósitos mostraram boa distribuição das partículas da concha na matriz de PEAD, nas diversas concentrações estudadas. As morfologias das superfícies de fratura dos compósitos mostraram uma larga distribuição do tamanho de partículas do pó da concha, o que já era esperado devido à sua larga distribuição de tamanho de partículas, conforme a análise granulométrica mostrada na Figura 2. Possivelmente, a boa distribuição das partículas na matriz foi causada durante o processo de mistura intensiva no misturador interno. Os aspectos morfológicos das superfícies de fratura dos compósitos são mostrados na Figura 5. De um modo geral, pode se observar que ocorreu ancoramento mecânico das partículas na matriz (indicado por "A" nas micrografias) como também a presença de vazios na matriz (indicado por "VM" nas micrografias) e em torno das partículas (indicado por "VP" nas micrografias), o que pode ter levado ao descolamento das partículas, refletindo o nível baixo de adesão entre a concha e o PEAD. Wang et al[15] também observaram deslocamento das partículas de hidroxiapatita a partir da matriz de polietileno. Renner et al [16] também observaram vazios em torno das partículas de carbonato de cálcio em compósitos de polipropileno. Estes resultados indicam a necessidade de fortalecer a adesão interfacial desses compósitos e nesse sentido alguns autores [5] têm sugerido a modificação da superfície da partícula de carbonato de cálcio com agentes de acoplamento do tipo titanato. O tamanho da partícula do pó da concha deve ser levado em consideração, como também a sua interação com a matriz, por serem fatores importantes no comportamento mecânico 
destes compósitos. Segundo Kar e Khatua[17], quanto menor o tamanho da partícula mais efetiva é a sua atuação como reforço para os compósitos. Outra consideração importante é a presença de aglomerados de partículas fracamente ligadas, principalmente no compósito 80:20(Figura 5c).
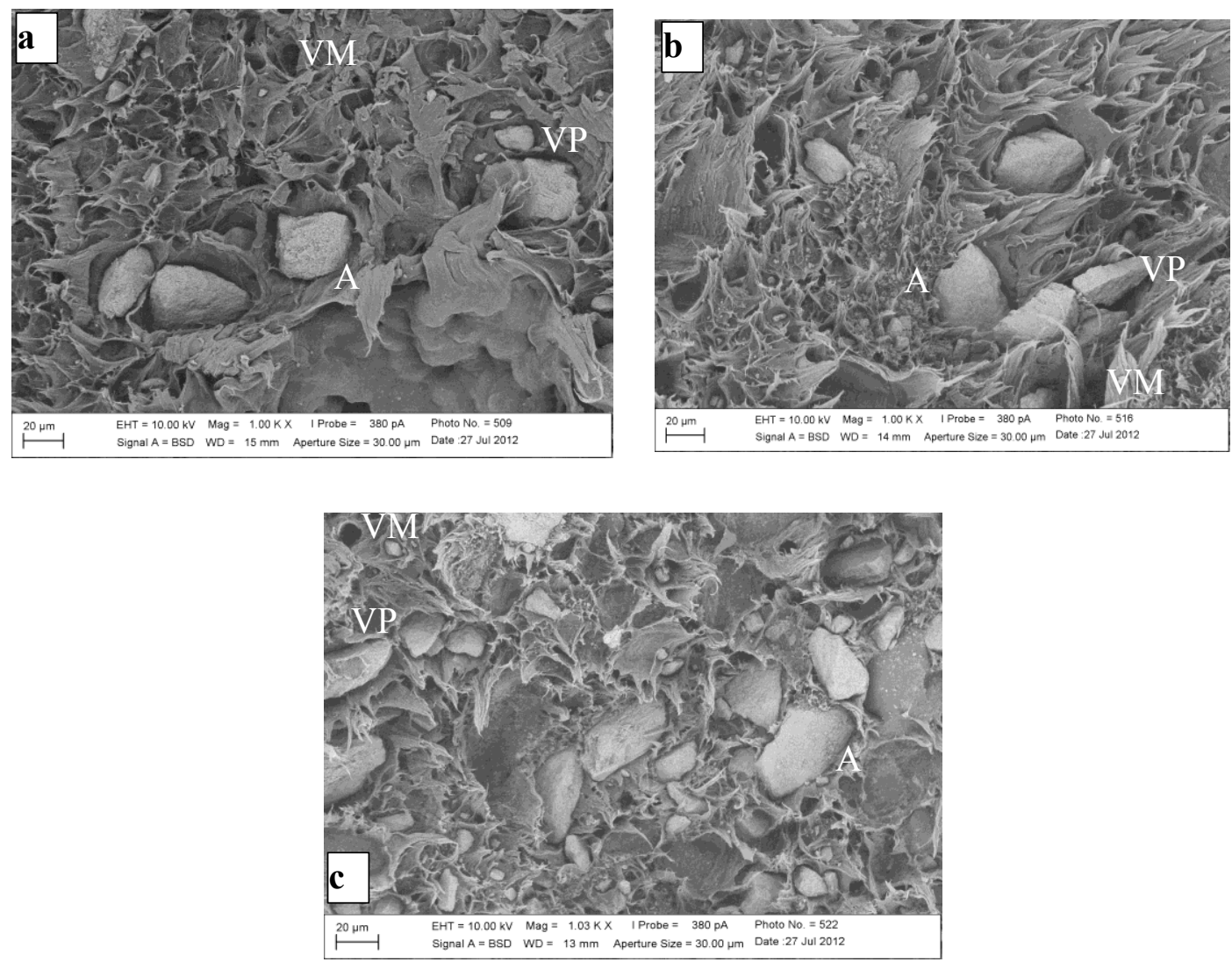

Figura 5: Micrografia dos compósitos PEAD/concha a) 95:5; b) 90:10 e c) 80:20.

\subsection{Análise Termo Dinâmico Mecânica (DMA)}

Utilizando os dados do DMA, um estudo das propriedades viscoelásticas de compósitos de PEAD com 5, 10 e $20 \%$ de concha é apresentado na faixa de temperatura de 30 a $100{ }^{\circ} \mathrm{C}$. O módulo de armazenamento (E') denota o máximo de energia armazenada no material durante a deformação. Quando a energia não é armazenada, se converte em calor por meio da fricção molecular. O módulo de perda (E") é essa energia dissipada como calor. A transição vítrea ( $\mathrm{Tg}$ ) é a temperatura onde o E" alcança um valor máximo. Abaixo da $\mathrm{Tg}$, a rigidez do polímero é relacionada com a mudança na energia elástica armazenada durante a deformação, sendo a mesma associada com deslocamentos pequenos das moléculas a partir de suas posições de equilíbrio.

Na Figura 6 são mostradas as curvas de E' para o PEAD puro e seus compósitos. Pode se observar que os compósitos apresentaram maior rigidez do que a matriz pura em toda a faixa de temperatura estudada (30 a $100{ }^{\circ} \mathrm{C}$ ), principalmente quando o conteúdo de concha foi aumentado para $20 \%$. Em todas as amostras, o aumento de $\mathrm{E}^{\prime}$ foi mais significativo na temperatura de $30^{\circ} \mathrm{C}$ e diminuiu com o aumento da temperatura. Pode-se sugerir que a concha atuou reforçando a matriz de PEAD. A presença de partículas atuando como reforço em matrizes poliméricas tem sido reportada por outros autores[15,17-21] usando zeolita, hidroxiapatita, fibras, talco, argilas, etc, sendo esse aumento da rigidez atribuído a maior transferência de força aplicada a matriz para as partículas [22-25]. Alguns autores[20,21,23] estudaram o efeito do tratamento superficial de partículas de celulose, partículas de talco com silano no módulo de armazenamento dos compósitos, obtendo melhoramento considerável, por exemplo, Abdelmouleh et al[20,21] atribuíram este efeito às interações entre o silano e os monômeros das resinas poliéster insaturado e epóxi. No presente estudo, os compósitos de PEAD/pó de concha exibiram melhoramento no $\mathrm{E}^{\prime}$ sem ter sido realizado nenhum tratamento superficial nas partículas da concha. 


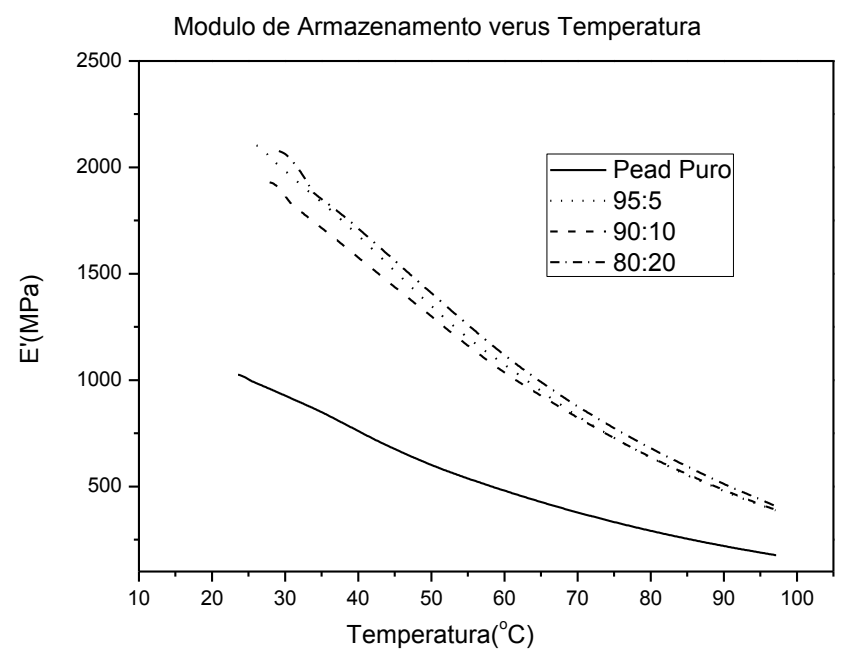

Figura 6: Módulo de Armazenamento, E’, do PEAD puro e dos seus compósitos.

As curvas do módulo de Perda (E") são mostrados na Figura 7. O formato dos picos de E" dos compósitos 95:5 e 90:10 foram similares ao da matriz. O compósito com 10\% de concha apresentou Tg deslocada em $6{ }^{\circ} \mathrm{C}$ em relação a Tg do PEAD puro( em torno de $60{ }^{\circ} \mathrm{C}$ ) e os valores máximos para o módulo de perda foram 201MPa e 108MPa, respectivamente. O aumento no $\mathrm{E}^{\prime \prime}$ dos compósitos mostra que os mesmos têm melhor habilidade para dissipar a energia vibracional, na forma de calor, do que a matriz pura de PEAD. Com relação ao aumento no valor de Tg apresentado pelo compósito 90:10, outros autores $[18,19,26]$ também observaram esse aumento com a inclusão de partículas rígidas em matrizes poliméricas, o que foi atribuído às interações entre as partículas e o polímero, eles sugeriram que ocorreu imobilização parcial das cadeias poliméricas e bloqueios físicos nas configurações moleculares nas vizinhanças das partículas. A hipótese desses autores foi baseada no estudo de Yim et al[27] que provaram que ocorreu a formação de uma camada mais rígida nas vizinhanças das partículas em compósitos particulados, devido a adsorção das moléculas do polímero na superfície das partículas. Portanto, a inibição da Tg no compósito 80:20 pode estar relacionada a imobilização molecular, como também a diminuição de volume livre da matriz de PEAD com presença de maior teor de partículas, conduzindo a maior fricção entre as próprias partículas, presentes nos aglomerados fracamente ligados nesse compósito (veja a Figura 5c).

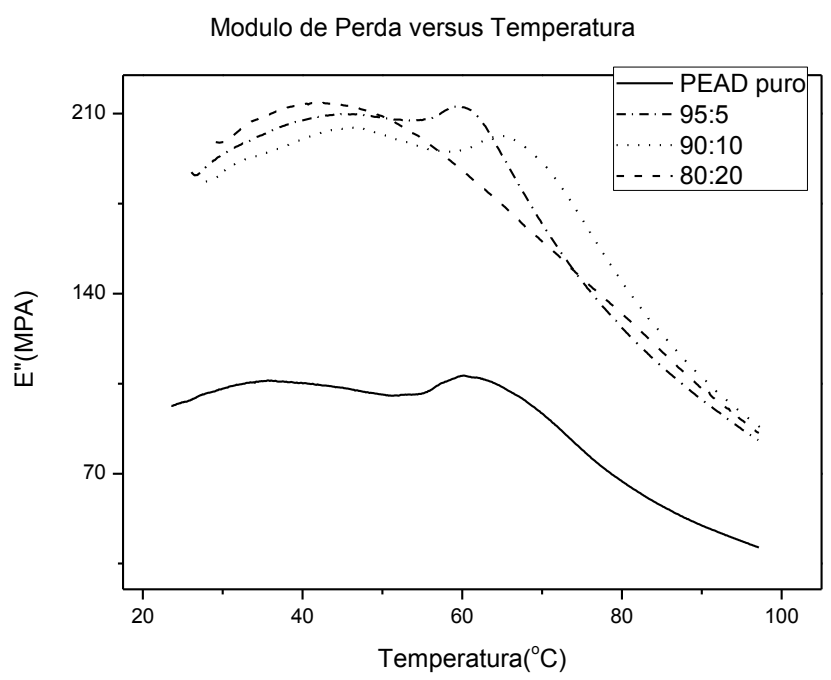

Figura 7: Módulo de Perda, E”, do PEAD puro e dos seus compósitos. 
$O$ tanঠ é a razão entre a energia dissipada ( $\left.E^{\prime \prime}\right)$ por ciclo pela energia armazenada (E') durante o ciclo. No contexto de engenharia, a capacidade alta de amortecimento $(\tan \delta)$ é essencial para reduzir o efeito de vibrações, tais como redução da amplitude de vibrações de ressonância para limites seguros, redução de ruídos, porém pode reduzir a estabilidade dimensional do material. Na Figura 8 são mostradas as curvas de $\tan \delta$ do PEAD e de seus compósitos. Pode se observar que o $\tan \delta$ aumentou com a adição do pó da concha, o que significa redução na capacidade de amortecimento dos compósitos. Para justificar essa redução, alguns autores[15,25] tem sugerido que se deve a limitação na mobilidade dos segmentos de cadeia da matriz e a diminuição do volume livre da fase amorfa viscoelástica da matriz, devido a maior interação entre as partículas e o polímero e consequente dissipação de energia. Este efeito foi mais intenso com a concentração de $20 \%$ da concha no compósito, não sendo observado o pico de Tan $\delta$, o que causou maior deterioração da capacidade de amortecimento da matriz de PEAD, por outro lado aumentou a estabilidade dimensional desse compósito. Similar resultado foi observado por Mukhopadhyay et al [26] ao utilizarem concentração mais alta de partículas de sílica na matriz da borracha de etileno acetato de vinila.

O efeito da adição de partículas rígidas a polímeros é diminuir sua capacidade de amortecimento. Nielsen e Lande [28] estimaram uma equação que avalia o efeito de cargas rígidas no amortecimento de polímeros, conforme mostrada a eq.2:

$$
\left(\frac{E^{\prime \prime}}{E^{\prime}}\right)_{c o m p}=\tan \delta_{c o m p}=\left(\tan \delta_{\text {matriz }} x \varphi_{\text {matriz }}\right)+\left(\tan \delta_{\text {part }} x \varphi_{\text {part }}\right)=\tan \delta_{\text {matriz }} x\left(1-\varphi_{\text {part }}\right)
$$

Onde: E' e E" são os módulos de perda e armazenamento, respectivamente, $\varphi_{\text {matriz }}$ e $\varphi_{\text {part }}$ são as frações da matriz e da partícula, respectivamente.

Desde que a o amortecimento da maior parte das partículas é muito baixo comparado ao amortecimento dos polímeros, o tan $\delta$ da partícula foi negligenciado na equação 2 e portanto não foi considerado nos cálculos. Essa equação sugere que a adição de partículas rígidas diminui o pico de dissipação de energia, devido ao decréscimo na fração de volume da matriz, esse decréscimo tem sido mostrado na literatura [22-24].

A partir da equação 2 e utilizando os dados da curva de $\tan \delta$ da matriz pura (Figura 8a), os valores teóricos de $\tan \delta$ dos compósitos foram calculados e são mostrados na Figura $8 b$. Esses valores foram obtidos em três temperaturas diferentes a 55,67 e $75^{\circ} \mathrm{C}$. Pode se observar que quanto maior a temperatura e a concentração do pó de concha mais a propriedade viscoelástica de amortecimento da matriz PEAD foi diminuída. Embora o compósito 80:20 não tenha apresentado pico de $\tan \delta$ ( Figura 8a), teoricamente pode se constatar este compósito foi o que apresentou maior redução na capacidade de amortecimento da matriz.

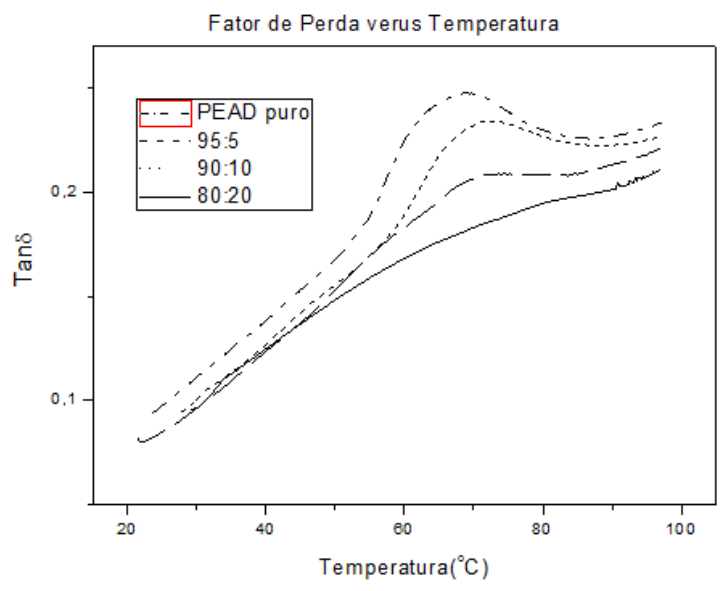

(a)

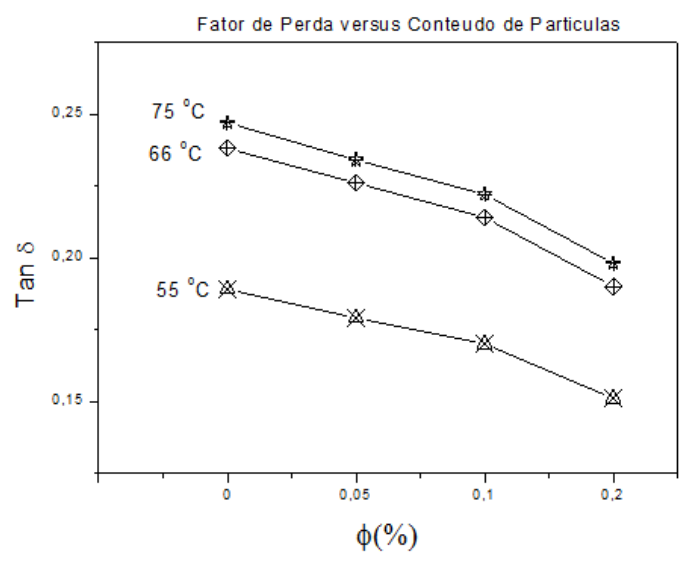

(b)

Figura 8: Tan $\delta$ do PEAD puro e dos seus compósitos : a) efeito da temperatura; b) efeito do conteúdo do pó de concha e da temperatura. 


\section{CONCLUSÃO}

Compósitos de PEAD com 5, 10 e 20\% em peso do pó de concha de molusco foram preparados. Foi constatado que a concha é constituída por mais de $90 \%$ de óxido de cálcio, com estrutura cristalina composta por de calcita e aragonita, polimorfos do carbonato de cálcio

A adição do pó da concha não influenciou nem a cristalinidade e nem a temperatura de fusão do PEAD nos compósitos. $\mathrm{Na}$ análise termogravimétrica, a adição da concha aumentou a temperatura de início da decomposição da matriz. O índice de fluidez dos compósitos foi inferior ao da matriz, reduzindo o fluxo e a processabilidade dos mesmos. A análise da morfologia mostrou que a adesão entre a concha e o PEAD foi fraca. O módulo de armazenamento dos compósitos foi maior do que o da matriz, o compósito 90:10 apresentou transição vítrea $6{ }^{\circ} \mathrm{C}$ mais alta do que a da matriz, já o compósito com $20 \%$ de concha não apresentou essa transição. A adição da concha diminuiu a capacidade de amortecimento do PEAD.

Portanto a adição do pó de concha ao PEAD resultou em compósitos com maior estabilidade térmica e dimensional sem deteriorar a cristalinidade da matriz polimérica. A partir da análise morfológica pode-se concluir que a moagem e a classificação granulométrica da concha devem ser bem controladas para evitar uma distribuição larga no tamanho de partículas e consequente aglomeração de partículas pequenas entre as de maior tamanho, comprometendo a interação interfacial dos compósitos. Portanto a utilização do pó de concha de molusco como reforço em compósitos poliméricos com PEAD pode ser promissora, amenizando os danos ambientais provenientes do seu descarte na região costeira de João Pessoa-PB.

\section{AGRADECIMENTOS}

A realização do presente trabalho só foi possível devido ao apoio financeiro do CNPQ por meio do Projeto Universal Proc. 476514/2010-0. Agradecemos ao Laboratório de Solidificação Rápida da UFPB, ao Laboratório de Materiais Compósitos da UFRN e ao Laboratório de Caracterização de Materiais da UFCG pelo apoio na realização das caracterizações.

\section{BIBLIOGRAFIA}

[1] TANNIRU, M., MISRA, R.D.K. "Reduced susceptibility to stress whitening during tensile deformation of calcium carbonate-reinforced high density polyethylene composites", Materials science \& engineering. A, Structural materials: properties, microstructure and processing, v. 424, pp. 53-70, May 2006.

[2] PALANIANDY, S., KADIR, N.A., JAAFAR, M. "Value adding limestone to filler grade through an ultra-fine grinding process in Jet Mill for use in plastic industries", Minerals engineering, v. 22, pp.695-703, June-Jully 2009.

[3] TANNIRU, M., MISRA, R.D.K., "On enhanced impact strength of calcium carbonate-reinforced highdensity polyethylene composites", Materials science \& engineering. A, Structural materials: properties, microstructure and processing, v. 405, pp. 178-193, Sep.2005.

[4] TANNIRU, M., MISRA, R.D.K., BERBRAND, K., et al., "The determining role of calcium carbonate on surface deformation during scratching of calcium carbonate-reinforced polyethylene composites", Materials science \& engineering. A, Structural materials: properties, microstructure and processing, v. 404, pp. 208220, Sep. 2005.

[5] GONZALEZ, J., ALBANO, C., ICHAZO, M., et al., "Effects of coupling agents on mechanical and morphological behavior of the PP/HDPE blend with two different CaCO3", European Polymer Journal, v. 38, 2465-2475, April 2002.

[6] WANG, C., XU, Y., YALAN LIU, Y., et al., "Synthesis and characterization of lamellar aragonite with hydrophobic property", Materials science \& engineering. C, Biomimetic materials, sensors and systems, v. 29, n.3, pp. 843-846, April 2009.

[7] ADDADI, S., WEINER, A. "Pavement of Pearl”, Nature, v. 389, pp. 912-915, 1997.

[8] KAMAT, S., SU, X., BALLARINI, R., et al., "Structural basis for the fracture toughness of the shell of the conch Strombus gigas", Nature, v. 405, pp. 1036-1040, Jun. 2000.

[9] HADAL, R.S., DASARI, A., ROHRMANN, J., et al., "Effect of wollastonite and talc on the micromechanisms of tensile deformation in polypropylene composites", Materials science \& engineering. A, Structural materials: properties, microstructure and processing, v. 372, pp. 296-315, May 2004.

[10] HADAL, R.S., MISRA, R.D.K., "The influence of loading rate and concurrent microstructural evolution in micrometric talc-and wollastonite - reinforced high isotactic polypropylene composites", Materials 
science \& engineering. A, Structural materials: properties, microstructure and processing, v. 374, pp. 374389, Jun. 2004.

[11] KREVELEN, D. W., Properties of Polymers, Elsevier, 1990.

[12] BEZERRA, U.T., ALMEIDA, F.L.P., SILVA, L.B., et al., "Production of filler aggregate from waste of bivalves molluscs shells", Proceedings of the 11th International Conference on Non-conventional Materials and Technologies (NOCMAT), n. 97, pp. 6-9, Sep. 2009.

[13] ALI SYED, M., RAMARAJ, B., AKHTAR, S., et al., "Development of Environmentally Friendly HighDensity Polyethylene and Turmeric Spent Composites: Physicomechanical, Thermal, and Morphological Studies", Journal of Applied Polymer Science, v. 118, n. 3, pp. 1204-1210, oct. 2010.

[14] LI, N.S, HUA, H.K., MEI, L.C., "Kinetic calculations for the thermal decomposition of calcium propionate under non-isothermal conditions", Chinese Science Bulletin, v.56, n.12, pp. 1278-1284, April 2011.

[15] WANG, M., JOSEPH, R., BONFIELD, W., "Hydroxyapatite-polyethylene composites for bone substitution: effects of ceramic particle size and morphology", Biomaterials, v.19, pp. 2357-2366, Jun. 1998.

[16] RENNER, K., YANG, M.S., MOCZO, J., et al., "Analysis of the debonding process in polypropylene model composites", European Polymer Journal, v.41, pp. 2520-2529, May 2005.

[17] SARKHEL, G., CHOUDHURY, A., "Dynamic Mechanical and Thermal Properties of PE-EPDM Based Jute Fiber Composites", Journal of Applied Polymer Science, v. 108, pp. 3442-3453, Jun. 2008.

[18] BLEACH, N.C., NAZHAT, S. N., TANNER, K. E., et al., "Effect of filler content on mechanical and dynamic mechanical properties of particulate biphasic calcium phosphate polylactide composites", Biomaterials, v.23, pp. 1579-1585, april 2002.

[19] NAZHAT, S.N., KELLOMAKI, M., TANNER, K.E., et al.," Dynamic mechanical characterisation of biodegradable compo-sites of hydroxyapatite and polylactides". J Biomed Mater Res (Appl Biomater), v. 58, pp.335-43, May 2001.

[20] ABDELMOULEH, M., BOUFI, S., BELGACEM, M. N., et al., "Modification of Cellulose Fibers with Functionalized Silanes: Effect of the Fiber Treatment on the Mechanical Performances of CelluloseThermoset Composites". Journal of Applied Polymer Science, v. 98, pp. 974-984, Dec. 2005.

[21] ABDELMOULEH, M., BOUFI, S., BELGACEM, M. N., et al., "Modification of cellulosic fibres with functionalised silanes: development of surface properties", International Journal Adhesion and Adhesives, v. 24, pp.43-54, Feb.2004.

[22] YUZAY, I.E., AURAS, R., SELKE, S., "Poly(lactic acid) and Zeolite Composites Prepared by Melt Processing: Morphological and Physical-Mechanical Properties", Journal of Applied Polymer Science, v. 115, pp.2262-2270, Feb 2010.

[23] HUDA, M.S., DRZAL, L. T., MOHANTY, A. K., et al., "The effect of silane treated- and untreated-talc on the mechanical and physico-mechanical properties of poly (lactic acid)/newspaper fibers/talc hybrid composites", Composites: Part B, V.38 pp.367-379, April 2007.

[24] LU, J., WANG, LAWR T., DRZAL, L. T., "Preparation and properties of microfibrillated cellulose polyvinyl alcohol composite materials", Composites: Part A, v. 39, pp.738-746, May 2008.

[25] LIU, Y., WANG, M., "Fabrication and Characteristics of Hydroxyapatite Reinforced Polypropylene as a Bone Analogue Biomaterial", Journal of Applied Polymer Science, v.106, pp. 2780-2790, Nov 2007.

[26] MUKHOPADHYAY, K., TRIPATHY, D.K., S. K., DE. "Dynamic Mechanical Properties of SilicaFilled Ethylene Vinyl Acetate Rubber", Journal of Applied Polymer Science, v. 48, pp.1089-1103, May 1993.

[27] YIM, A., CHAHAL, R.S., ST. PIERRE, L.E., "The Effect of Polymer-Filler Interaction Energy on the T'g of Filled Polymers". Journal of Colloid and Interface Science, v. 43, n. 3, pp. 583-590, June 1973.

[28] NIELSEN, L. E., LANDEL, R. F., Mechanical Properties of Polymers and Composites, Marcel Dekker, 1994. 